Check for updates

Cite this: RSC Adv., 2017, 7, 47288

Received 14th September 2017 Accepted 29th September 2017

DOI: 10.1039/c7ra10237b

rsc.li/rsc-advances

\section{An ionic liquid-organic solvent biphasic system for efficient production of 5-hydroxymethylfurfural from carbohydrates at high concentrations}

\begin{abstract}
Qidong Hou, (D) Weizun Li, Meinan Zhen, Le Liu, Yu Chen, Qian Yang, Fang Huang, Shiqiu Zhang and Meiting Ju*

5-Hydroxymethylfurfural (HMF) is an important intermediate in the utilization of lignocellulosic biomass. Ionic liquid 1-ethyl-3-methylimidazolium bromide (EMIMBr) containing a dissolved $\mathrm{SnCl}_{4}$ as catalyst was found to be capable to convert carbohydrates into HMF, as an alternative to the chromium-based catalytic system. Based on this, a biphasic system consisting of glycol dimethyl ether or dimethyl carbonate as the extraction phase, and $\mathrm{EMIMBr} / \mathrm{SnCl}_{4}$ in combination with partial dissolved organic solvent as the reaction phase was developed for the production of HMF from carbohydrates (80 wt\% with respect to the ionic liquid) at high concentrations. The biphasic system could obtain HMF yields (49.0-65.7\%) comparable to or slightly higher than the monophasic system. The biphasic system could not only considerably displace ionic liquids with organic solvents, but also promote the separation of HMF from the reaction system owing to the excellent extraction ability and low boiling point of the organic solvents.
\end{abstract}

\section{Introduction}

The utilization of lignocellulosic biomass to replace fossil fuel resources could provide one of the most promising ways to solve the current aggravating resource crisis and environmental problems. ${ }^{1}$ To achieve this goal, various possible routes have been explored to produce alternative fuels, chemicals and materials using renewable biomass as feedstock. ${ }^{2}$ In 2004, a range of biomass-derived chemicals which can be converted into value-added products via further conversion were recognized as platform chemicals. ${ }^{3}$ Among the platform chemicals, 5-hydroxymethylfurfural (HMF), a triple dehydration product of $\mathrm{C}_{6}$ monosaccharides, has risen in prominence as key starting material of biofuels, fine chemicals and polymeric materials for either drop in or new applications. ${ }^{4-7}$ For instance, one of the oxidation products of HMF, 2,5-furandicarboxylic acid (FDCA) can serve as an alternative building block to produce polyethylene terephthalate (PEF)., ${ }^{2,8} \mathrm{PEF}$ has been reported to have superior physical properties to PTT (polyethylene terephthalate), a polymer produced at 50 megatons a year as an industrial commodity. ${ }^{9}$ Therefore, the synthesis of HMF from lignocellulose is a critical step to obtain more value-added products from biomass. ${ }^{10}$

Tianjin Engineering Research Center of Biomass Solid Waste Resources Technology, College of Environmental Science and Engineering, Nankai University, 94 Weijin Street, Tianjin 300071, PR China. E-mail: jumeit@nankai.edu.cn; Tel: +86 13820988813
Although numerous approaches have been used to convert carbohydrates into HMF, the large-scale production of HMF is still not practical due to some economic and technical barriers.,11 Many studies have achieved highly efficient conversion of fructose into HMF, but the relative low abundance of fructose in nature results in the high cost of fructose. ${ }^{12-25}$ Production of HMF from glucose, the most abundant component in lignocellulosic biomass, is economically more attractive than from fructose. ${ }^{26}$ Nevertheless, although numerous approaches have been attempted, only a few reaction systems could selectively convert glucose into HMF. ${ }^{27}$ One kinds of catalytic systems are based on biphasic system that could concurrently extract HMF into an organic phase immediately after its formation in the reactive phase. ${ }^{4,28-31}$ In these biphasic systems, several solid catalysts, such as phosphated $\mathrm{TiO}_{2}, \mathrm{Nb}-$ doped tungstite and $\mathrm{SnPO}_{4}$ were reported to be capable of obtaining relatively high HMF yields. ${ }^{32-36}$ Besides, the combination of Lewis acid and Brønsted acid was also used for the conversion of glucose into HMF. ${ }^{37-41}$ However, selective conversion of glucose into HMF in these reaction systems generally requires unrealistic reaction conditions, including high temperature, low glucose concentration, high loading of solid catalyst and high concentration of salt to deliver HMF into the extraction phase. ${ }^{4,32,35,42}$ Even when homogeneous Lewis acid, such as $\mathrm{AlCl}_{3}$ was used as catalyst, high temperature and low glucose concentration are still needed to obtain a high HMF selectivity and glucose conversion. ${ }^{43-45}$ Another outstanding catalytic system is metal halides dissolved in ionic liquid. ${ }^{46}$ HMF yields near 70\% were obtained from the ionic liquid 1- 
ethyl-3-methyl-imidazolium chloride (EMIMCl) using $\mathrm{CrCl}_{2}$ and $\mathrm{CrCl}_{3}$ as catalysts. ${ }^{47}$ Using $\mathrm{CrCl}_{3}$ as catalyst, an ionic liquidorganics-water ternary biphasic system which could convert high-concentration glucose $(80 \mathrm{wt} \%$ with respect to the ionic liquid) into HMF with high yield was developed. ${ }^{27} \mathrm{CrCl}_{2}$ and $\mathrm{CrCl}_{3}$ were also demonstrated to be efficient to convert carbohydrates into HMF in several other ionic liquids, dimethyl acetamide and aqueous biphasic system. ${ }^{26,48-50}$ However, the large scale application of chromium salts may induce serious environmental pollutions because of their toxicity. ${ }^{51,52}$ Therefore, it is highly desirable but challenging to develop chromium-free reaction system for the production of HMF from carbohydrates at high concentration. ${ }^{\mathbf{4 6}}$

To achieve efficient HMF production from carbohydrates at high concentrations, a catalytic system based on ionic liquidorganic solvent biphasic system was developed. A series of ionic liquids and metal salts were screened to displace the widely investigated chromium based catalytic system. The influences of water, organic solvent, reaction condition and substrate on the HMF yields were also investigated to enhance the HMF production.

\section{Experimental}

\subsection{Materials and reagents}

Fructose (99\%), glucose (99\%), 5-hydroxymethylfurfural (99\%), $\mathrm{SnCl}_{4} \cdot 5 \mathrm{H}_{2} \mathrm{O}, \mathrm{SnCl}_{2} \cdot 2 \mathrm{H}_{2} \mathrm{O}, \mathrm{CrCl}_{3} \cdot 6 \mathrm{H}_{2} \mathrm{O}, \mathrm{AlCl}_{3} \cdot 6 \mathrm{H}_{2} \mathrm{O}, \mathrm{MgCl}_{2}-$ $\cdot 6 \mathrm{H}_{2} \mathrm{O}, \mathrm{ZnCl}_{2}, \mathrm{ZrOCl}_{2}, \mathrm{FeCl}_{3}, \mathrm{MnCl}_{2}$, glycol dimethyl ether (GDE, 99\%), dimethyl carbonate (DMC, 99\%), tetrahydrofuran (THF, 99\%), methyl isobutyl ketone (MIBK, 99\%) and dimethyl sulphoxide (DMSO, 99\%) were purchased from Tianjin Heowns Biochem LLC (Tianjin, China). Ionic liquids, including AMIMCl (1-allyl-3-methylimidazolium chloride), EMIMCl, BMIMCl, EMIMBr, 1-butyl-3-methylimidazolium bromide (BMIMBr), 1-ethyl-3-methylimidazolium tetrafluoroborate $\left(\mathrm{EMIMBF}_{4}\right)$, 1-butyl-3-methylimidazolium tetrafluoroborate $\left(\mathrm{BMIMBF}_{4}\right)$ was purchased from Shanghai Chengjie Chemical Reagent Co. Ltd., China. Another $\mathrm{EMIMBF}_{4}$ products was obtained from Lanzhou zhongkekaite Co. Ltd., China. All the other chemical reagents were purchased from commercial sources in China and used as received.

\subsection{Catalytic tests}

Known amounts of carbohydrate, metal salt and IL (or the biphasic system consisting of IL and organic solvent) were added to a thick-walled glass vial. The metal slats loading of $10 \mathrm{~mol} \%$ with respect to monosaccharide were used in all cases. The glass vial was sealed with polytetrafluoroethylene plug and then heated in an oil bath, with the mixture being stirred by a magnetic stir bar in the reactor at $850 \mathrm{rpm}$. The mixture was stirred at a fixed temperature for desired time. After the desired reaction time elapsed, the reactor was cooled to room temperature with cold water bath, and diluted with water (for pure IL) or methanol (for the biphasic system). The sample was then filtered with a $0.45 \mu \mathrm{m}$ polytetrafluoroethylene filter membrane to remove the insoluble solid. The concentration of HMF was measured with a high-performance liquid chromatography (HPLC) equipped with a diode array detector (DAD) at $284 \mathrm{~nm}$. The concentration of glucose and fructose was measured with a HPLC systems equipped with an evaporative light scattering detector (SofTA, ELSD Model 300s) and a Xtimate® Sugar-Ca analytical column $(7.8 \times 300 \mathrm{~mm}, 5 \mu \mathrm{m})$. The column oven temperature was set as $80{ }^{\circ} \mathrm{C}$, and the mobile phase was ultrapure water at a flow rate of $0.50 \mathrm{ml} \mathrm{min}^{-1}$. The catalytic performance of the reaction system was evaluated via observing the conversion of glucose and the selectivity and yield of the products. To evaluate the partition of HMF between the reaction phase and the extracting phase, the reaction phase and the extracting phase were separated using a syringe and needle. After separation, the reaction phase and the extracting phase were diluted and measured according to the above procedure, respectively. All results were replicated at least three times, and reproducibility of sugar conversion, HMF yield and HMF selectivity were within $3 \%$ standard deviation. The recycling experiment was performed for five cycles using glucose as substrate. After each reaction, the reaction phase and the extracting phase was separated using a syringe and needle. The remaining $\mathrm{HMF}$ in the reaction phase was repeatedly extracted with GDE and then the mixture containing EMIMBr, $\mathrm{SnCl}_{4}$ and humins was obtained. The mixture was diluted with water and then filtrated to remove humins. $\mathrm{EMIMBr}$ and $\mathrm{SnCl}_{4}$ was recovered together after removing water by distilling.

\section{Results and discussion}

\subsection{Effect of catalysts on the dehydration of glucose}

Various metal salts were tested for the dehydration of glucose to $\mathrm{HMF}$ at $100{ }^{\circ} \mathrm{C}$ for $3 \mathrm{~h}$ in ionic liquid EMIMBr. As shown in Fig. $1, \mathrm{CrCl}_{3}, \mathrm{SnCl}_{2}$ and $\mathrm{SnCl}_{4}$ exhibited high glucose conversion

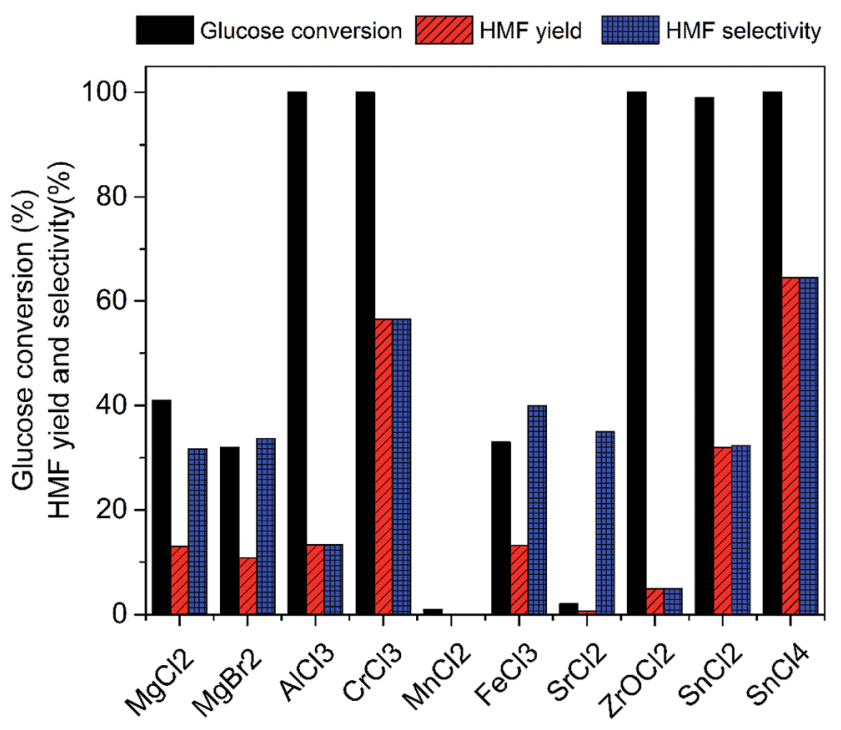

Fig. 1 Effect of catalysts on the dehydration of glucose into HMF in $\mathrm{EMIMBr}$. Reaction conditions: $100 \mathrm{mg}$ of glucose was dissolved in $1000 \mathrm{mg}$ of EMIMBr; molar ratio of catalyst : glucose $=1: 10 ; 100{ }^{\circ} \mathrm{C}$; $3 \mathrm{~h}$. 
and $\mathrm{HMF}$ yield. In contrast, $\mathrm{MgCl}_{2}, \mathrm{MgBr}_{2}, \mathrm{MnCl}_{2}, \mathrm{FeCl}_{3}, \mathrm{SrCl}_{2}$ showed inefficient glucose conversion and low HMF yield. Although $\mathrm{AlCl}_{3}$ and $\mathrm{ZrOCl}_{2}$ showed high glucose conversions approaching $100 \%$, the yields of HMF was low due to the poor selectivity. Remarkable insoluble solids were observed when the reaction mixtures were diluted with water, suggesting large amount of glucose was transformed into humins.

Subsequently, $\mathrm{SnCl}_{2}, \mathrm{SnCl}_{4}$ and $\mathrm{CrCl}_{3}$ were chosen as three representative metal chlorides to investigate the preliminary reaction kinetics of glucose dehydration in detail. As shown in Fig. 2, the best result was attained with $\mathrm{SnCl}_{4}$ which gives a high HMF yield of $64.5 \%$ with a glucose conversion approaching $100 \%$. Both the HMF yield and selectivity obtained with $\mathrm{SnCl}_{4}$ were higher than that obtained with $\mathrm{CrCl}_{3}$ during the whole reaction process. The HMF yield obtained with $\mathrm{CrCl}_{3}$ using EMIMBr as solvent was comparable to previous reports obtained with $\mathrm{CrCl}_{3}$ and $\mathrm{CrCl}_{2}$ weather using BMIMCl or EMIMCl as reaction medium. ${ }^{27,47}$ Therefore, our results demonstrated that the $\mathrm{SnCl}_{4} / \mathrm{EMIMBr}$ system is comparable to or slightly more capable than the $\mathrm{CrCl}_{3}$ and $\mathrm{CrCl}_{2}$ based reaction system for the conversion of glucose into HMF. Compared with $\mathrm{CrCl}_{3}$ and $\mathrm{CrCl}_{2}, \mathrm{SnCl}_{4}$ is more cheaper and has much lower toxicity. ${ }^{53}$

\subsection{Effect of substrate on the synthesis of $\mathbf{H M F}$}

The EMIMBr/SnCl${ }_{4}$ system can be extended to catalyze the conversion of other sugars, including fructose, sucrose, maltose, cellobiose and starch. Compared with glucose, the conversion of fructose was faster with a higher HMF yield (Fig. 3). It's reported previously that dissolution of $\mathrm{SnCl}_{4}$ in water produce a solution with $\mathrm{pH}$ of 1.8 , as is remarkably lower than the $\mathrm{pH}$ of the aqueous solution of other Lewis acidic metal chlorides. ${ }^{43} \mathrm{SnCl}_{4}$ was also reported to be active to catalyze the hydrolysis of sucrose, maltose, cellobiose, waste cooked rice and bread crust into monosaccharide in aqueous solution. ${ }^{54}$

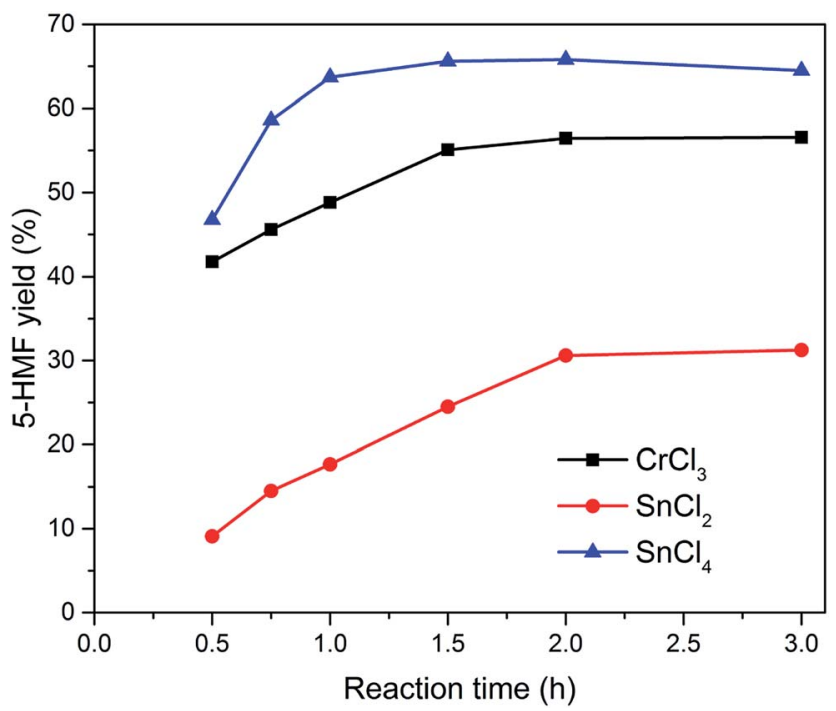

Fig. 2 Effect of reaction time on the yield of HMF. Reaction conditions: $100 \mathrm{mg}$ of glucose was dissolved in $1000 \mathrm{mg}$ of EMIMBr; molar ratio of catalyst : glucose $=1: 10 ; 100^{\circ} \mathrm{C}$.

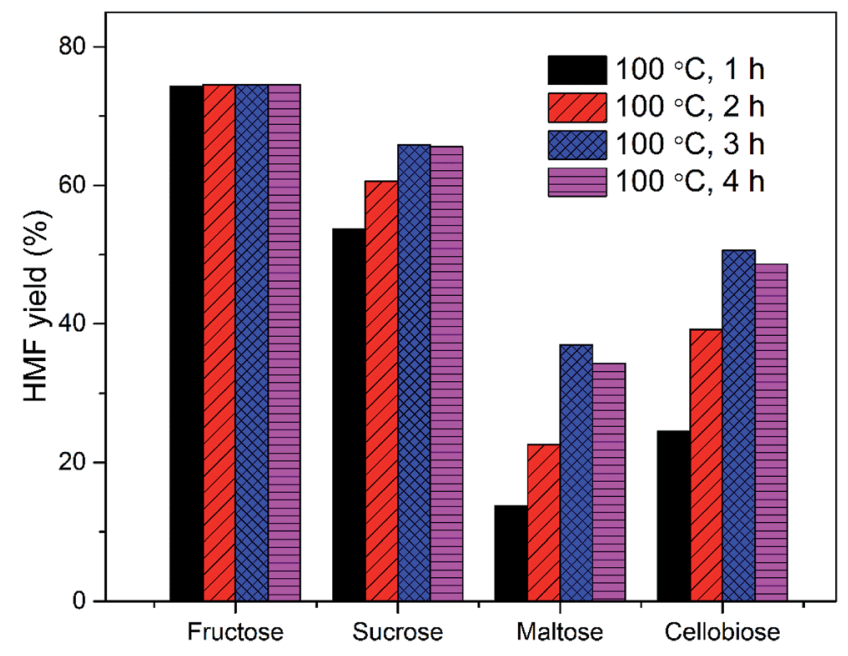

Fig. 3 HMF yield from fructose, glucose, sucrose, maltose, cellobiose and starch. Reaction conditions: $100 \mathrm{mg}$ of sugar was dissolved in $1000 \mathrm{mg}$ of $\mathrm{EMIMBr}$; molar ratio of $\mathrm{SnCl}_{4}:$ monosaccharide $=1: 10$; $100{ }^{\circ} \mathrm{C}$.

These results indicated that some Brønsted acid is formed from the reaction system when $\mathrm{SnCl}_{4}$ contacts with water. When sucrose was used as the feedstock, a disaccharide consisting of equimolar glucose and fructose, the HMF yield was slightly higher than that obtained from glucose, indicating that both glucose and fructose was released from sucrose and subsequently dehydrated into HMF. When maltose and cellobiose were tested, the maximum HMF yields were 37.0 and $50.6 \%$, respectively, as are lower than that obtained from glucose. When starch was used as feedstock, the HMF yield was only $8.9 \%$ after $2 \mathrm{~h}$ at $100{ }^{\circ} \mathrm{C}$ (Fig. 4). The HMF yield from starch was improved to $43.4 \%$ when the reaction was performed at $120{ }^{\circ} \mathrm{C}$ for $3 \mathrm{~h}$, demonstrating that starch can be depolymerized into glucose and dehydrated into HMF through one-pot reaction.

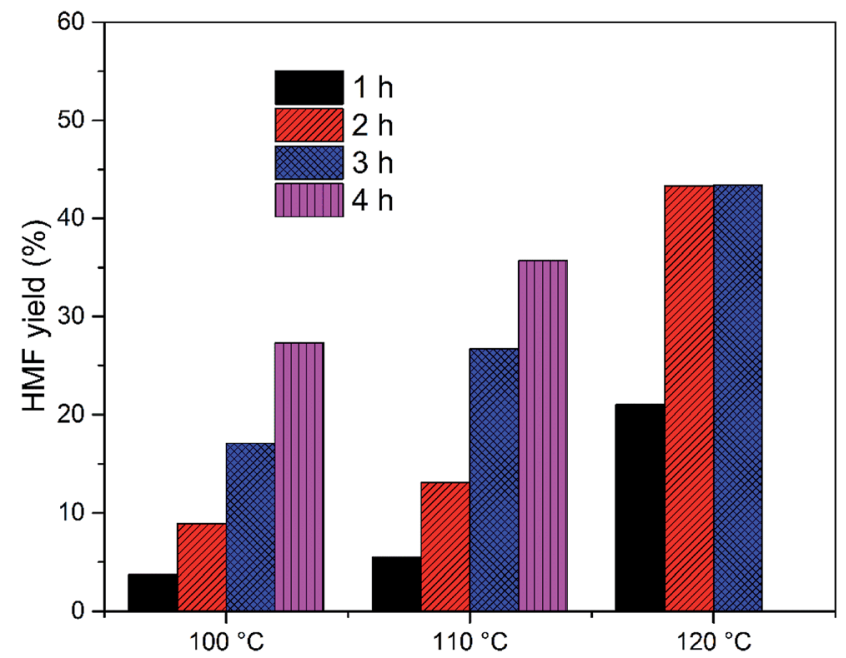

Fig. 4 HMF yield from starch. Reaction conditions: $100 \mathrm{mg}$ of starch was dissolved in $1000 \mathrm{mg}$ of $\mathrm{EMIMBr}$; molar ratio of $\mathrm{SnCl}_{4}:$ glucose $=$ $1: 10$. 
The influence of the initial sugar concentration on the HMF yield in the $\mathrm{EMIMBr} / \mathrm{SnCl}_{4}$ system was investigated. When the loading of glucose increased from $10 \mathrm{wt} \%$ to $20 \mathrm{wt} \%$, the yield was almost unchanged (Fig. 5). However, the further increase of the glucose concentration resulted in the gradually decrease of the HMF yield. When the reaction mixtures were diluted with water, large amounts of humins were observed, suggesting that the decrease of HMF yield was mainly attributed to the accelerated side-reactions. It should be noted that the HMF yield can still reach $40 \%$ even at a glucose loading up to $100 \mathrm{wt} \%$ (glucose/EMIMBr weight ratio $=1$ ). The HMF yield was remarkable higher than that obtained with the $\mathrm{BMIMCl} / \mathrm{CrCl}_{3}$ system under similar conditions, demonstrating that the $\mathrm{EMIMBr} / \mathrm{SnCl}_{4}$ system is more active than the widely used $\mathrm{BMIMCl} / \mathrm{CrCl}_{3}$ system. ${ }^{27}$

\subsection{Effect of solvents on the dehydration of glucose into HMF}

The effect of solvents on the conversion of glucose into HMF using $\mathrm{SnCl}_{4}$ as catalyst was also investigated. As shown in Table 1, the HMF yield from BMIMBr was comparable to that obtained with EMIMBr. When the anion was $\mathrm{Cl}^{-}, \mathrm{BF}_{4}{ }^{-}$or $\mathrm{I}^{-}$, remarkable lower yields of HMF were obtained. Wrigstedt et al. investigated the effect of salts on the $\mathrm{CrCl}_{3}$-catalyzed glucose dehydration to HMF in aqueous media. ${ }^{49}$ They found that the bromide anions could enhance the fructose dehydration step in aqueous media, also leading to higher HMF yields than the corresponding chloride slats. A previous study reported that the highest yield of HMF was attained using $\mathrm{EMIMBF}_{4}$ as solvent. ${ }^{53}$ However, our experiment showed that the HMF yield is just $6.8 \%$ when $\mathrm{EMIMBF}_{4}$ was used as the reaction medium under the same condition. Two commercial available $\mathrm{EMIMBF}_{4}$ from different sources were used to preclude the possible effect of the impurity in ionic liquid. It was also observed that the reaction

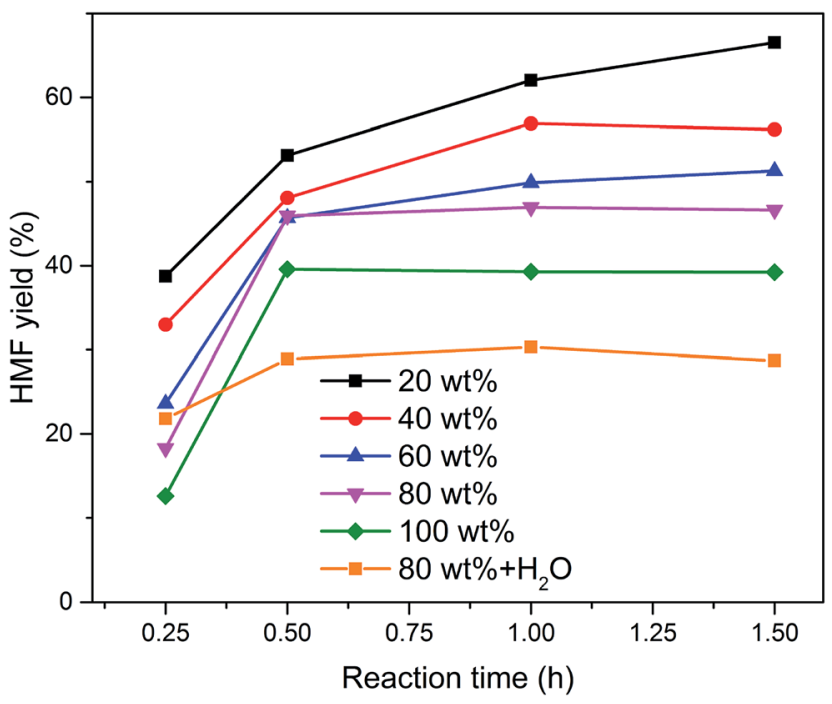

Fig. 5 Effect of sugar loading on the synthesis of HMF from glucose. Reaction conditions: $1000 \mathrm{mg}$ EMIMBr; molar ratio of $\mathrm{SnCl}_{4}$ : glucose $=1: 10 ; 100{ }^{\circ} \mathrm{C}$.
Table 1 Effect of solvents on the dehydration of glucose into $\mathrm{HMF}^{a}$

\begin{tabular}{|c|c|c|c|c|}
\hline Catalyst & Solvent & Temperature $\left({ }^{\circ} \mathrm{C}\right)$ & Time (h) & HMF yield (\%) \\
\hline $\mathrm{SnCl}_{4}$ & BMIMBr & 100 & 3 & 62.1 \\
\hline $\mathrm{SnCl}_{4}$ & EMIMCl & 100 & 3 & 19.8 \\
\hline $\mathrm{SnCl}_{4}$ & BMIMCl & 100 & 3 & 21.6 \\
\hline $\mathrm{SnCl}_{4}$ & $\mathrm{EMIMBF}_{4}$ & 100 & 0.5 & 13.5 \\
\hline $\mathrm{SnCl}_{4}$ & $\mathrm{EMIMBF}_{4}$ & 100 & 1 & 6.8 \\
\hline $\mathrm{SnCl}_{4}$ & $\mathrm{EMIMBF}_{4}$ & 80 & 1 & 6.6 \\
\hline $\mathrm{SnCl}_{4}$ & $\mathrm{EMIMBF}_{4}$ & 80 & 2 & 9.3 \\
\hline $\mathrm{SnCl}_{4}$ & $\mathrm{BMIMBF}_{4}$ & 100 & 1 & 0.9 \\
\hline $\mathrm{SnCl}_{4}$ & DMSO & 100 & 1 & 22.1 \\
\hline $\mathrm{SnCl}_{4}$ & GDE & 100 & 3 & 18.6 \\
\hline $\mathrm{SnCl}_{4}$ & GDE & 120 & 1 & 22.7 \\
\hline $\mathrm{CrCl}_{3}$ & GDE & 100 & 3 & 16.1 \\
\hline
\end{tabular}

${ }^{a}$ Reaction conditions: $100 \mathrm{mg}$ of glucose was dissolved in $1000 \mathrm{mg}$ of solvent; molar ratio of catalyst : glucose $=1: 10$.

mixture became dark brown quickly, suggesting the formation of humins. When the reaction temperature was reduced to $80{ }^{\circ} \mathrm{C}$, the HMF yield could be improved to $9.3 \%$, as is still significantly lower than the previous report. D'Anna et al. also reported that $\mathrm{HMF}$ is unstable in pure $\mathrm{EMIMBF}_{4} \cdot{ }^{55}$ Besides, it's observed glycol dimethyl ether (GDE) could also severed as a potential solvent for the conversion of sugars, obtaining HMF yields of 18.6 and $16.1 \%$ with $\mathrm{SnCl}_{4}$ and $\mathrm{CrCl}_{3}$, respectively. The HMF yield from GDE was lower than that from EMIMBr but comparable to that obtained with DMSO, one of the most widely used solvent for sugar dehydration reactions. Besides, it was observed that adding water to EMIMBr lead to the decrease of HMF yield (Fig. 5). In summary, BMIMBr, EMIMBr and GDE are promising reaction medium for $\mathrm{SnCl}_{4}$ to catalyze the conversion of glucose into HMF.

To understand the role of EMIMBr in the conversion of sugars into HMF, the conversion of fructose in EMIMBr in the absence of catalyst was also investigated. As expected, EMIMBr was very effective for the direct conversion of fructose into HMF without utilizing any other additive or catalyst. Under the optimized conditions, nearly $100 \%$ conversion of fructose with a $92 \%$ yield of HMF was obtained. Li et al. performed the direct conversion of fructose to HMF in a series of ionic liquids. ${ }^{56}$ They found that BMIMBr, $\mathrm{HPyBr}$ give both high fructose conversion and HMF yield, while other ionic liquids, including BMIMCl, BMIMI and $\mathrm{C}_{6} \mathrm{MIMBF}_{4}$ are not as effective as BMIMBr. Zhao et al. reported that the HMF yield from the dehydration of fructose in EMIMCl is $40 \%$ with a fructose conversion approaching $60 \%$ at $100{ }^{\circ} \mathrm{C}$ for $3 \mathrm{~h}^{47}$ Moreover, it should be noted that EMIMBr could catalyze the conversion of fructose at high loading. As shown in Fig. 6, increasing the initial fructose concentration had a negative impact on the HMF yield. Even so, a HMF yield approaching $40 \%$ could be obtained from EMIMBr even at a sugar loading as high as $100 \mathrm{wt} \%$ with respect to EMIMBr (glucose/EMIMBr weight ratio $=1$ ). In summary, EMIMBr and BMIMBr showed much higher activity for the direct dehydration of fructose into HMF than other ionic liquids.

The reaction rate of glucose dehydration into HMF in EMIMBr in the presence $\mathrm{SnCl}_{4}$ was considerably higher than 


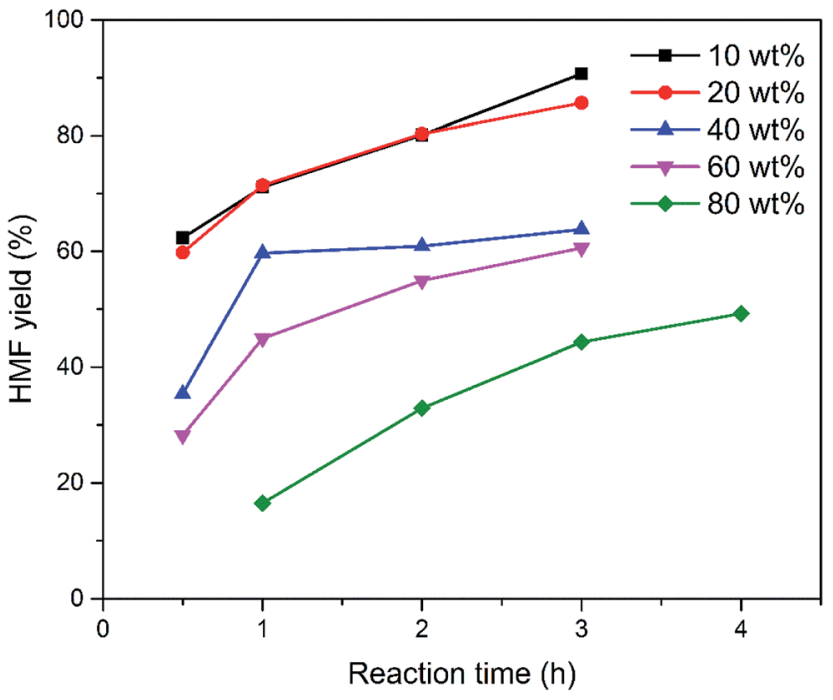

Fig. 6 Effect of sugar loading on the synthesis of HMF from fructose in $\mathrm{EMIMBr}$ without additional catalyst. Reaction conditions: $1000 \mathrm{mg}$ EMIMBr; $100{ }^{\circ} \mathrm{C}$.

that of fructose dehydration in pure EMIMBr, suggesting that $\mathrm{SnCl}_{4}$ play an important role not only in the isomerization of glucose into fructose, but also in the dehydration of fructose to HMF. The isomerization of glucose into fructose is mainly catalyzed by Lewis acid, while the dehydration of fructose into HMF is mainly catalyzed by Brønsted acid. ${ }^{57}$ The superior activity of $\mathrm{SnCl}_{4}$ for glucose isomerization could be attributed to anhydrous environment in EMIMBr. Although $\mathrm{SnCl}_{4}$ could serve as the catalyst for the conversion of glucose into HMF in aqueous solution, the activity is low even at $170{ }^{\circ} \mathrm{C} \cdot{ }^{\mathbf{4 3 , 5 8}}$ Moliner et al. reported that tetra-coordinated $\mathrm{Sn}^{4+}$ within zeolites framework are highly active for the isomerization of glucose into fructose in water at $110{ }^{\circ} \mathrm{C}$, while $\mathrm{SnCl}_{4}$ can't catalyze this reaction at the same temperature. ${ }^{59}$ BermejoDeval et al. reported that extra framework tin sites located within the hydrophobic channels of zeolite beta can isomerize glucose to fructose in both water and methanol solvent, while $\mathrm{SnO}_{2}$ particles located at external zeolite crystal surfaces or supported on amorphous silica catalyze isomerization in methanol but not in water. ${ }^{60}$ These results suggested that the contact with bulk water inhibits the catalytic activity of $\mathrm{Sn}^{4+}$ sites for glucose isomerization. Our studies also indicated that the addition of water has an adverse impact on the glucose dehydration in BMIMBr (Fig. 5). Since the water concentration (less than $1 \%$, formed in the sugar dehydration process) was low, the $\mathrm{Sn}^{4+}$ from the $\mathrm{BMIMBr} / \mathrm{SnCl}_{4}$ system could catalyze the isomerization of glucose in a similar way with the tetracoordinated $\mathrm{Sn}^{4+}$ located in the hydrophobic channels of zeolite beta and the $\mathrm{SnO}_{2}$ located in anhydrous environment. Some Brønsted acid will be produced when $\mathrm{SnCl}_{4}$ contacts with the formed water and the formed Brønsted acid could accelerate the dehydration of fructose. Therefore, the higher dehydration rate of glucose in the $\mathrm{SnCl}_{4} / \mathrm{EMIMBr}$ system was attributed to the synergistic effect between EMIMBr and $\mathrm{SnCl}_{4}$.
3.3.1. Conversion of carbohydrates into HMF in the biphasic system. Although the $\mathrm{EMIMBr} / \mathrm{SnCl}_{4}$ system is efficient for the conversion of carbohydrates into HMF, the high cost of ionic liquids will be a major barrier for its large scale application. One strategy to solve this problem is designing an ionic liquid-organic solvent biphasic system which could suppress the degradation of the formed HMF via continuously extracting HMF into the organic phase during the reaction. Previous studies have demonstrated that tetrahydrofuran (THF), methyl isobutyl ketone (MIBK) and dimethyl carbonate (DMC) are effective to extract HMF from the aqueous solution., ${ }^{\mathbf{4 4 , 6 1}}$ The influence of these organic solvents, including GDE, DMC, MIBK and THF, on the conversion of fructose in EMIMBr in the absence of additional catalyst were tested. As shown in Fig. 7, all the organic solvents have a negative impact on the catalytic activity of BMIMBr. In the tested organic solvents, GDE has the lowest negative impact on the catalytic activity.

Subsequently, the organic solvents were combined with $\mathrm{EMIMBr} / \mathrm{SnCl}_{4}$ to form biphasic system for the catalytic conversion of glucose at high concentrations. As shown in Fig. 8, all the biphasic systems exhibited high catalytic activities in converting glucose even at a glucose loading as high as $80 \mathrm{wt} \%$. However, the HMF yield varies in a wide range depending on the organic solvents used. The maximal HMF yields of $58.7 \%$ and $58.4 \%$ were achieved from the GDE/ EMIMBr and DMC/EMIMBr system, respectively. The HMF yields from these two biphasic systems were remarkable higher than that obtained from pure EMIMBr with the same sugar loading. The other organic solvent-EMIMBr systems were close to or even less effective than pure EMIMBr. All the four organic solvents can form two phases with the EMIMBr and partially extract the formed HMF from EMIMBr to the organic phase during the reaction process. The amount of HMF in the extraction phase corresponds to HMF yields of 33.1, 37.5, 19.0 and $27.2 \%$ for GDE, DMC, MIBK and THF, respectively (Fig. 9).

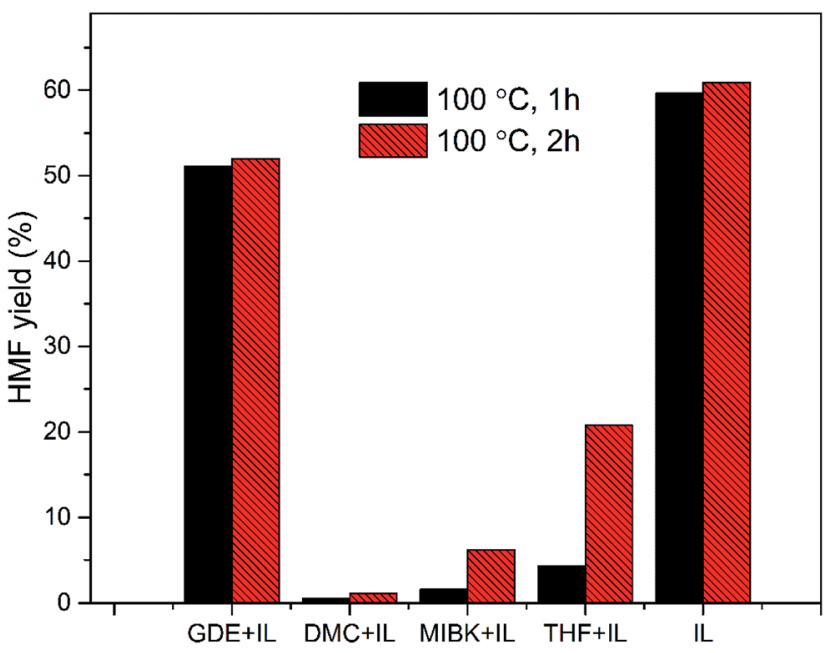

Fig. 7 Effect of organic solvents on the synthesis of HMF from fructose in the biphasic system without additional catalyst. Reaction conditions: $100 \mathrm{mg}$ fructose; $250 \mathrm{mg} \mathrm{EMIMBr}, 2 \mathrm{ml}$ organic solvent, $100^{\circ} \mathrm{C}$. 


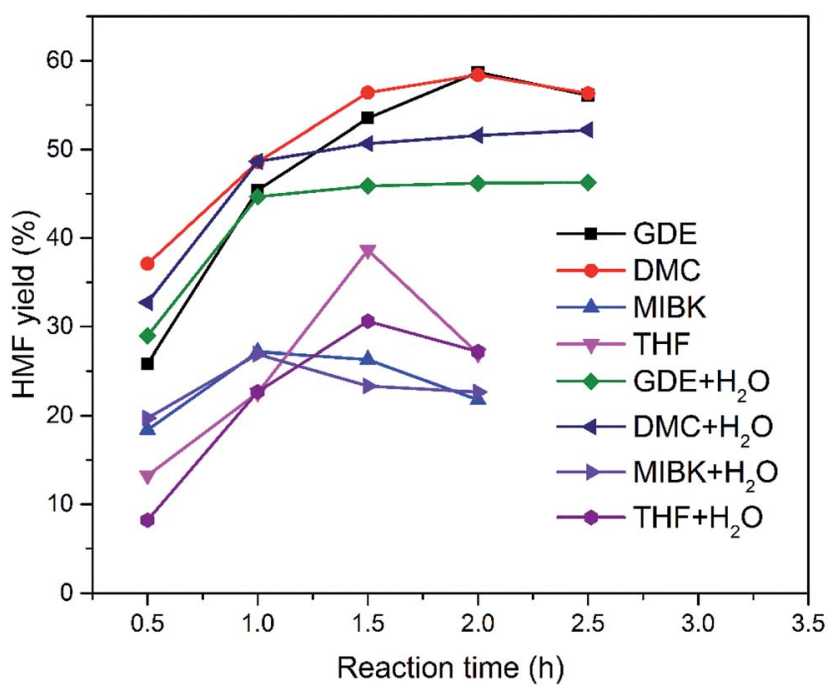

Fig. 8 Effect of organic solvents on the yield of HMF in the biphasic system at a glucose loading of $80 \mathrm{wt} \%$. Reaction conditions: $250 \mathrm{mg}$ $\mathrm{EMIMBr} ; 2 \mathrm{ml}$ organic solvent; $200 \mathrm{mg}$ glucose; molar ratio of $\mathrm{SnCl}_{4}:$ glucose $=1: 10 ; 100{ }^{\circ} \mathrm{C}$.

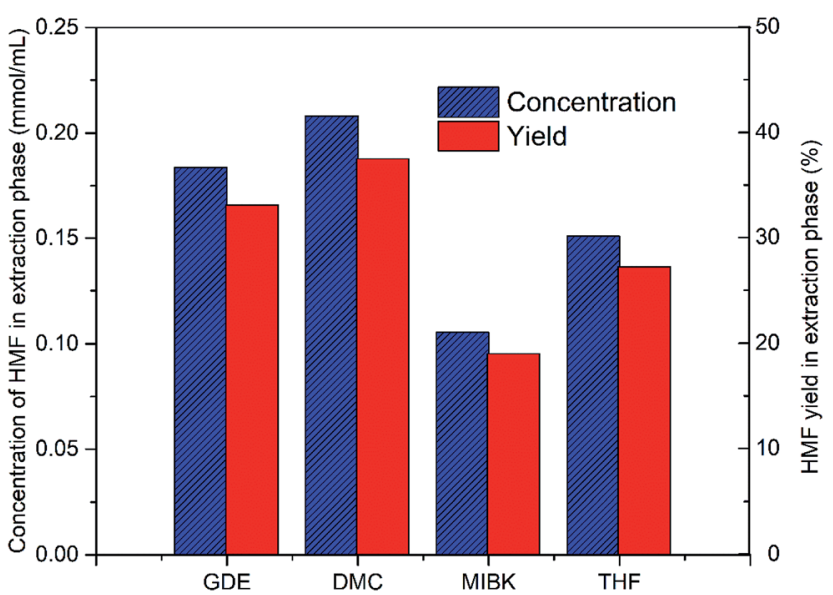

Fig. 9 HMF distribution in the extraction phase when glucose was converted in the biphasic system. Reaction conditions: $250 \mathrm{mg}$ $\mathrm{EMIMBr} ; 2 \mathrm{ml}$ organic solvent; $200 \mathrm{mg}$ glucose; molar ratio of $\mathrm{SnCl}_{4}:$ glucose $=1: 10 ; 100{ }^{\circ} \mathrm{C}$.

In the EMIMBr/DMC system, as high as $64.2 \%$ of the formed HMF was transferred from the reaction phase to the extraction phase, indicating that DMC has excellent extraction efficiency. As discussed above, DMC has a negative impact on the catalytic activity of EMIMBr for fructose dehydration (Fig. 7). Therefore, although DMC had a better extraction performance than GDE, the final yield of HMF from the DMC/EMIMBr system was comparable to GDE/EMIMBr. The addition of water had a detrimental impact on the HMF yield, as is different from the $\mathrm{GDE} / \mathrm{BMIMCl} / \mathrm{CrCl}_{3}$ system where appropriate amount of water is helpful for the production of HMF. ${ }^{27}$

In previous study, $\mathrm{SnCl}_{4}$ was also used for the production of HMF from glucose at $170{ }^{\circ} \mathrm{C}$ using a biphasic system consisting of $\mathrm{NaCl}$ aqueous solution and alkylphenol solvent. ${ }^{43}$ Compared with this system, our reaction system has many advantages. First, the loading of sugar in our work ( $10 \mathrm{wt} \%$ with respect to the total weight of the reaction system) was remarkable higher than that used in the previous biphasic system (1.7 wt\%). Second, the reaction temperature in our work is low, as will reduce the energy consumption. In addition, GDE and DMC can be used as extracting solvent for the separation of HMF from the reaction phase since the boiling point of $\operatorname{GDE}\left(83^{\circ} \mathrm{C}\right)$ and DMC $\left(90{ }^{\circ} \mathrm{C}\right)$ are significantly lower than that of HMF. Although alkylphenol solvent was reported to be effective to extract HMF from the aqueous solution, the separation of HMF and alkylphenol solvent is rather difficult due to their similar boiling points. ${ }^{43}$

As an alternative to the chromium-based catalytic system, the GDE/EMIMBr/SnCl ${ }_{4}$ system is also effective for the conversion of other sugars. The HMF yields from fructose, sucrose, maltose, cellobiose and starch were 63.5, 65.7, 49.0, 62.4 and $51.2 \%$, respectively (Fig. 10). In view of the total weight of GDE and EMIMBr, the sugar loading used in the biphasic system was approaching $10 \mathrm{wt} \%$. Therefore, a comparison between the GDE/EMIMBr system with an $80 \mathrm{wt} \%$ sugar loading (with respect to ionic liquid) and the monophasic EMIMBr with a $10 \mathrm{wt} \%$ sugar loading was performed. For monosaccharides, including glucose and fructose, the HMF yields from the GDE/ EMIMBr system were slightly lower than that obtained with monophasic EMIMBr and meanwhile a slightly longer reaction time was required to obtain the maximal HMF yield. This result was a combination of factors which influence the dehydration reaction. As stated above, the substitution of EMIMBr with GDE could not only reduce the catalytic activity of EMIMBr for fructose dehydration, but also reduce the degradation of HMF by extracting HMF to the organic phase. At the same time, the conversion of sugar into HMF with lower selectivity could also occur in pure GDE in the presence $\mathrm{SnCl}_{4}$ (Table 1). For disaccharides and polysaccharide, including sucrose, maltose,

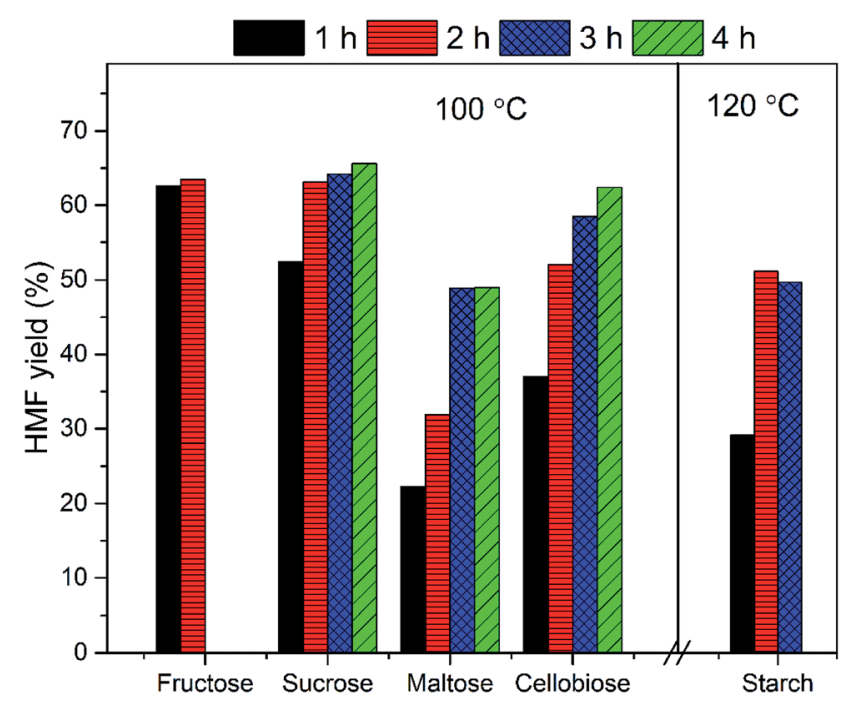

Fig. 10 Effect of substrate on the yield of HMF from the biphasic system. Reaction conditions: $250 \mathrm{mg} E M I M B r ; 2 \mathrm{ml} \mathrm{GDE} ; 200 \mathrm{mg}$ sugar; molar ratio of $\mathrm{SnCl}_{4}$ : monosaccharide $=1: 10$. 
cellobiose and starch, the HMF yields from the GDE/EMIMBr system were higher than that obtained with monophasic EMIMBr. The improved HMF yield from the EMIMBr/GDE system may be attributed to the relative low release rete of sugars from this system. Due to the high viscosity of highconcentration sugar solution, the hydrolysis rate of disaccharide and polysaccharide in EMIMBr/GDE was lower than in EMIMBr, as suppress the side reactions between sugars and HMF, thus resulting in the higher yield of HMF. It was also observed that starch can't be completely dissolved in EMIMBr/ GDE at the initial stage of the reaction due to the low usage of EMIMBr. The complete dissolution of starch was gradually achieved with the reaction proceeded. The HMF yield $(51.2 \%)$ obtained with the EMIMBr/GDE system from starch was remarkably higher than that obtained with the monophasic EMIMBr $(43.3 \%)$, also supporting that the slight inhibition of hydrolysis is helpful to improve the final yield of HMF. The HMF yield from EMIMBr/GDE system was also higher than a previous reports using $\mathrm{CrCl}_{2}$ as catalyst. ${ }^{62}$

To study the recyclability and stability of the EMIMBr/GDE/ $\mathrm{SnCl}_{4}$ system, a recycling experiment was performed using glucose as substrate for five cycles. After each reaction, EMIMBr and $\mathrm{SnCl}_{4}$ was recovered together. The recovered EMIMBr and $\mathrm{SnCl}_{4}$ were combined with GDE and then reused for the conversion of glucose into HMF. As shown in Fig. 11, after five recycles the conversion of fructose and the HMF yield are 98.0\% and $56.3 \%$, respectively. This result indicates that the catalytic system can be reused with a slight loss of catalytic activity.

Since the EMIMBr/GDE system could tolerate various sugar substrates at high concentrations, this system may be more profitable than monophasic ionic liquid for the production of HMF in actual biorefinery process. In practice, efficient and selective production of glucose from lignocellulosic biomass is particularly challenging by either chemical process or biochemical process due to the difficulty of separating cellulose

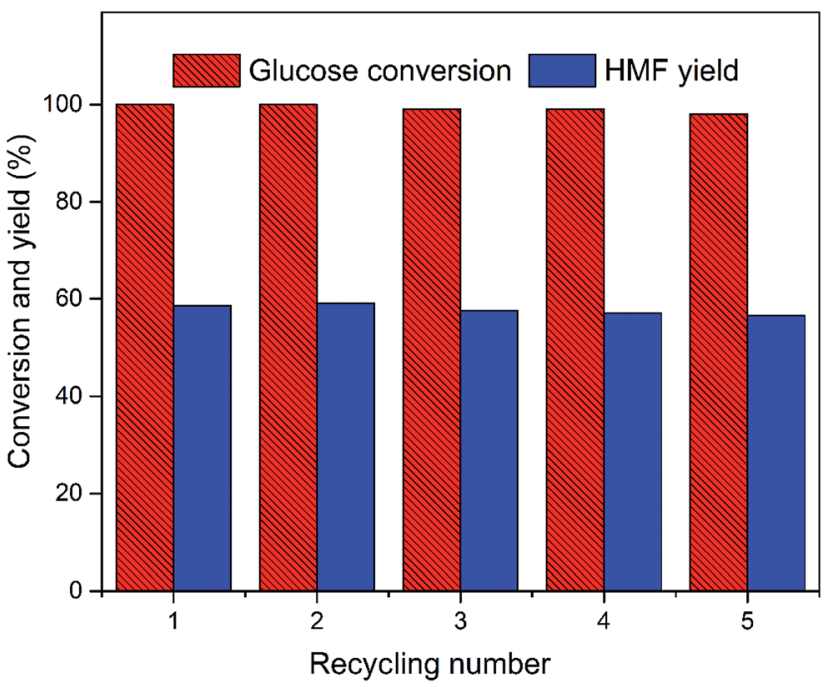

Fig. 11 Recycling of the catalytic system. Reaction conditions: $250 \mathrm{mg}$ EMIMBr; $2 \mathrm{ml} \mathrm{GDE} ; 200 \mathrm{mg}$ glucose; molar ratio of $\mathrm{SnCl}_{4}$ : glucose = $1: 10 ; 100{ }^{\circ} \mathrm{C} ; 2 \mathrm{~h}$. from biomass and the recalcitrance of cellulose. ${ }^{\mathbf{6 3} 64}$ Harsh reaction conditions, such high temperature and concentrated mineral acid could overcome the recalcitrance of crystalline cellulose, but the degradation of the produced sugars into unfavorable by-products also occur in these conditions. ${ }^{26,63}$ Compared with pure glucose, obtaining water-soluble oligosaccharides is easy to achieve through either acid-catalyzed hydrolysis, enzymatic saccharification or mechanocatalysis. ${ }^{63-66}$ At the same time, the attainable sugar yields can be maximized and the energy consumption can be reduced under relative mild condition. Since the EMIMBr/GDE system is suitable for the conversion of various sugar substrates, the oligosaccharides obtained at relative mild condition may be well suited for production of HMF using this reaction system.

More work is still required to further improve this system for economically favorable application on large scale. In practice, the use of ionic liquids as reaction medium for the conversion of carbohydrate into HMF is not universally applicable and any change of the composition and structure of ionic liquid, catalyst and co-solvent may impact the conversion of carbohydrate. ${ }^{26,46}$ To solve these problems, low-cost solvent systems, especially biomass derived ionic liquids or deem eutectic solvents (DESs) should be developed and investigated for this conversion as an alternative to the expensive imidazolium-based ionic liquids. ${ }^{67,68}$ Meanwhile, new strategy for the recovery and reuse of ionic liquids and catalyst should be developed to reduce environmental pollutions. In addition, although many efforts have been devoted to the one-pot conversion of cellulose into HMF, the yield and selectivity of HMF are low in most cases, even under harsh reaction conditions. ${ }^{69-73}$ Therefore, the cascade of the hydrolysis of cellulose and the dehydration of glucose into HMF should be further studied to achieve the onepot conversion of cellulose and lignocellulosic biomass into HMF by chromium-free reaction system.

\section{Conclusion}

A series of solvents and metal salts were screened to develop chromium-free reaction system for the production of HMF from glucose. EMIMBr/SnCl${ }_{4}$ was demonstrated as an efficient reaction system for the conversion of carbohydrates to HMF. Based on EMIMBr/SnCl 4 , a biphasic system containing GDE or DMC as the extraction phase was developed for the production of HMF from carbohydrates at high concentrations. Up to 58.7, $63.5,65.7,49.0,62.4$ and $51.2 \%$ of HMF yields were obtained from glucose, fructose, sucrose, maltose, cellobiose and starch even at a very high sugar concentration $(80 \mathrm{wt} \%$ with respect to the ionic liquid). Except for considerably displacing ionic liquids with organic solvents, the biphasic system could also promote the separation of HMF from ionic liquid owing to the excellent extraction ability for HMF and low boiling point of the organic solvents.

\section{Conflicts of interest}

There are no conflicts to declare. 


\section{Acknowledgements}

This work was supported by the Project supported by the National Science Foundation for Young Scientists of China (51708301), Research Projects in the Science \& Technology Pillar Program of Tianjin, China (16YFXTSF00030), Research Projects in the Science \& Technology Program of Jinnan District Tianjin, China (2015JNKW0005), Research Projects in the Science \& Technology Pillar Program of Tianjin, China (15JCTPJC63300) and Science Foundation of Tianjin, China (17JCZDJC39500).

\section{References}

1 A. Farrán, C. Cai, M. Sandoval, Y. Xu, J. Liu, M. J. Hernáiz and R. J. Linhardt, Chem. Rev., 2015, 115, 6811-6853.

2 I. Delidovich, P. J. C. Hausoul, L. Deng, R. Pfützenreuter, M. Rose and R. Palkovits, Chem. Rev., 2016, 116, 1540-1599.

3 R. A. Sheldon, Green Chem., 2014, 16, 950-963.

4 B. Saha and M. M. Abu-Omar, Green Chem., 2014, 16, 24-38. 5 J. Li, Y. Jing, C. Liu and D. Zhang, New J. Chem., 2017, 41, 8714-8720.

6 K. V. S. Ranganath, M. Sahu, M. Shaikh, P. K. Gavel, K. K. Atyam, S. Khilari and P. Das, New J. Chem., 2016, 40, 4468-4471.

7 S. Jia, X. He and Z. Xu, RSC Adv., 2017, 7, 39221-39227.

8 S. Siankevich, G. Savoglidis, Z. Fei, G. Laurenczy, D. T. L. Alexander, N. Yan and P. J. Dyson, J. Catal., 2014, 315, 67-74.

9 A. Banerjee, G. R. Dick, T. Yoshino and M. W. Kanan, Nature, 2016, 531, 215-219.

10 Z. Xue, M. G. Ma, Z. Li and T. Mu, RSC Adv., 2016, 6, 9887498892.

11 M. Han, X. Liu, G. Huang, Y. Liu and S. Ji, RSC Adv., 2016, 6, 47890-47896.

12 Y. Peng, Z. Hu, Y. Gao, D. Yuan, Z. Kang, Y. Qian, N. Yan and D. Zhao, ChemSusChem, 2015, 8, 3208-3212.

13 Q. Hou, W. Li, M. Ju, L. Liu, Y. Chen and Q. Yang, RSC Adv., 2016, 6, 104016-104024.

14 X. Tong, M. Li, N. Yan, Y. Ma, P. J. Dyson and Y. Li, Catal. Today, 2011, 175, 524-527.

15 E. L. S. Ngee, Y. Gao, X. Chen, T. M. Lee, Z. Hu, D. Zhao and N. Yan, Ind. Eng. Chem. Fundam., 2014, 53, 14225-14233.

16 Z. Hu, Y. Peng, Y. Gao, Y. Qian, S. Ying, D. Yuan, S. Horike, N. Ogiwara, R. Babarao, Y. Wang, N. Yan and D. Zhao, Chem. Mater., 2016, 28, 2659-2667.

17 Y. Wang, C. M. Pedersen, Y. Qiao, T. Deng, J. Shi and X. Hou, Carbohydr. Polym., 2015, 115, 439-443.

18 Z. Zhang, B. Liu and Z. Zhao, Carbohydr. Polym., 2012, 88, 891-895.

19 G. Raveendra, M. Surendar and P. S. Sai Prasad, New J. Chem., 2017, 41, 8520-8529.

20 H. Zhang, Y. Cheng, H. Yuan, Y. Wang and Z.-H. Ma, Catal. Sci. Technol., 2017, DOI: 10.1039/c7cy01340j.

21 H. Tang, N. Li, F. Chen, G. Li, A. Wang, Y. Cong, X. Wang and T. Zhang, Green Chem., 2017, 19, 1855-1860.

22 J. Dai, L. Zhu, D. Tang, X. Fu, J. Tang, X. Guo and C. Hu, Green Chem., 2017, 19, 1932-1939.
23 H. Han, H. Zhao, Y. Liu, Z. Li, J. Song, W. Chu and Z. Sun, RSC Adv., 2017, 7, 3790-3795.

24 F. Tao, H. Song and L. Chou, RSC Adv., 2011, 1, 672-676.

25 X. Qi, M. Watanabe, T. M. Aida and R. L. Smith Jr, ChemSusChem, 2009, 2, 944-946.

26 J. B. Binder and R. T. Raines, J. Am. Chem. Soc., 2009, 131, 1979-1985.

27 J. Zhou, Z. Xia, T. Huang, P. Yan, W. Xu, Z. Xu, J. Wang and Z. C. Zhang, Green Chem., 2015, 17, 4206-4216.

28 Y. Sun, P. Liu and Z. Liu, Carbohydr. Polym., 2016, 142, 177182.

29 D. Gupta, E. Ahmad, K. K. Pant and B. Saha, RSC Adv., 2017, 7, 41973-41979.

30 M. Li, W. Li, Y. Lu, H. Jameel, H. M. Chang and L. Ma, RSC Adv., 2017, 7, 14330-14336.

31 W. Li, Z. Xu, T. Zhang, G. Li, H. Jameel, H. Chang and L. Ma, BioResources, 2016, 11, 5839-5853.

32 A. Dutta, D. Gupta, A. K. Patra, B. Saha and A. Bhaumik, ChemSusChem, 2014, 7, 925-933.

33 L. Atanda, A. Shrotri, S. Mukundan, Q. Ma, M. Konarova and J. Beltramini, ChemSusChem, 2015, 8, 2907-2916.

34 L. Atanda, S. Mukundan, A. Shrotri, Q. Ma and J. Beltramini, ChemCatChem, 2015, 7, 781-790.

35 C. Yue, G. Li, E. A. Pidko, J. J. Wiesfeld, M. Rigutto and E. J. M. Hensen, ChemSusChem, 2016, 9, 2421-2429.

36 M. Hattori, K. Kamata and M. Hara, Phys. Chem. Chem. Phys., 2017, 19, 3688-3693.

37 T. Zhang, W. Fan, W. Li, Z. Xu, H. Xin, M. Su, Y. Lu and L. Ma, Energy Technol., 2016, 5, 747-755.

38 Y. Shen, Y. Zhang, Y. Chen, Y. Yan, J. Pan, M. Liu and W. Shi, Energy Technol., 2016, 4, 600-609.

39 H. Xin, T. Zhang, W. Li, M. Su, S. Li, Q. Shao and L. Ma, RSC Adv., 2017, 7, 41546-41551.

40 T. Zhang, W. Fan, W. Li, Z. Xu, H. Xin, M. Su, Y. Lu and L. Ma, Energy Technol., 2017, 5, 747-755.

41 Y. Lu, Z. Sun and M. Huo, RSC Adv., 2015, 5, 30869-30876.

42 W. Li, T. Zhang, H. Xin, M. Su, L. Ma, H. Jameel, H.-m. Chang and G. Pei, RSC Adv., 2017, 7, 27682-27688.

43 Y. J. Pagán-Torres, T. Wang, J. M. R. Gallo, B. H. Shanks and J. A. Dumesic, ACS Catal., 2012, 2, 930-934.

44 J. Tang, L. Zhu, X. Fu, J. Dai, X. Guo and C. Hu, ACS Catal., 2017, 256-266.

45 J. J. Wang, Z. C. Tan, C. C. Zhu, G. Miao, L. Z. Kong and Y. H. Sun, Green Chem., 2016, 18, 452-460.

46 M. E. Zakrzewska, E. Bogel-Łukasik and R. Bogel-Łukasik, Chem. Rev., 2011, 111, 397-417.

47 H. Zhao, J. E. Holladay, H. Brown and Z. C. Zhang, Science, 2007, 316, 1597-1600.

48 L. Hu, Y. Sun and L. Lin, Ind. Eng. Chem. Fundam., 2012, 51, 1099-1104.

49 P. Wrigstedt, J. Keskiväli, M. Leskelä and T. Repo, ChemCatChem, 2015, 7, 501-507.

50 S. Siankevich, Z. Fei, R. Scopelliti, P. G. Jessop, J. Zhang, N. Yan and P. J. Dyson, ChemSusChem, 2016, 9, 2089-2096.

51 A. Herbst and C. Janiak, New J. Chem., 2016, 40, 7958-7967.

52 J. M. J. M. Ravasco, J. A. S. Coelho, S. P. Simeonov and C. A. M. Afonso, RSC Adv., 2017, 7, 7555-7559. 
53 S. Hu, Z. Zhang, J. Song, Y. Zhou and B. Han, Green Chem., 2009, 11, 1746-1749.

54 I. K. M. Yu, D. C. W. Tsang, A. C. K. Yip, S. S. Chen, Y. S. Ok and C. S. Poon, Bioresour. Technol., 2016, 219, 338-347.

55 F. D'Anna, S. Marullo, P. Vitale, C. Rizzo, P. Lo Meo and R. Noto, Appl. Catal., A, 2014, 482, 287-293.

56 Y. N. Li, J. Q. Wang, L. N. He, Z. Z. Yang, A. H. Liu, B. Yu and C. R. Luan, Green Chem., 2012, 14, 2752-2758.

57 T. Wang, M. W. Nolte and B. H. Shanks, Green Chem., 2014, 16, 548-572.

58 K. R. Enslow and A. T. Bell, Catal. Sci. Technol., 2015, 5, 28392847.

59 M. Moliner, Y. Román-Leshkov and M. E. Davis, Proc. Natl. Acad. Sci. U. S. A., 2010, 107, 6164-6168.

60 R. Bermejo-Deval, R. Gounder and M. E. Davis, ACS Catal., 2012, 2, 2705-2713.

61 A. Dibenedetto, M. Aresta, L. Di Bitonto and C. Pastore, ChemSusChem, 2016, 9, 118-125.

62 S. Siankevich, Z. Fei, R. Scopelliti, G. Laurenczy, S. Katsyuba, N. Yan and P. J. Dyson, ChemSusChem, 2014, 7, 1647-1654.

63 J. S. Luterbacher, J. M. Rand, D. M. Alonso, J. Han, J. T. Youngquist, C. T. Maravelias, B. F. Pfleger and J. A. Dumesic, Science, 2014, 343, 277-280.
64 L. Hu, L. Lin, Z. Wu, S. Zhou and S. Liu, Appl. Catal., B, 2015, 174-175, 225-243.

65 M. Käldström, N. Meine, C. Farès, F. Schüth and R. Rinaldi, Green Chem., 2014, 16, 3528-3538.

66 S. Mateo, J. G. Puentes, S. Sánchez and A. J. Moya, Carbohydr. Polym., 2013, 93, 416-423.

67 A. M. Socha, R. Parthasarathi, J. Shi, S. Pattathil, D. Whyte, M. Bergeron, A. George, K. Tran, V. Stavila, S. Venkatachalam, M. G. Hahn, B. A. Simmons and S. Singh, Proc. Natl. Acad. Sci. U. S. A., 2014, 111, 3587-3595.

68 A. M. Da Costa Lopes and R. Bogel-Lukasik, ChemSusChem, 2015, 8, 947-965.

69 G. Zi, Z. Yan, Y. Wang, Y. Chen, Y. Guo, F. Yuan, W. Gao, Y. Wang and J. Wang, Carbohydr. Polym., 2015, 115, 146-151.

70 F. Yang, Y. Li, Q. Zhang, X. Sun, H. Fan, N. Xu and G. Li, Carbohydr. Polym., 2015, 131, 9-14.

71 F. Tao, H. Song, J. Yang and L. Chou, Carbohydr. Polym., 2011, 85, 363-368.

72 T. W. Ching, V. Haritos and A. Tanksale, Carbohydr. Polym., 2017, 157, 1794-1800.

73 J. Pan, Y. Mao, H. Gao, Q. Xiong, F. Qiu, T. Zhang and X. Niu, Carbohydr. Polym., 2016, 143, 212-222. 